\title{
BMJ Open Evaluation protocol to assess maternal and child health outcomes using administrative data: a community health worker home visiting programme
}

\author{
Samantha Sabo, ${ }^{1}$ Matthew Butler, ${ }^{2}$ Kelly McCue (D) , ${ }^{1}$ Patrick Wightman, ${ }^{3}$ \\ Vern Pilling, ${ }^{4}$ Martín Celaya, ${ }^{5}$ Sara Rumann ${ }^{5}$
}

To cite: Sabo S, Butler M, McCue $\mathrm{K}$, et al. Evaluation protocol to assess maternal and child health outcomes using administrative data: a community health worker home visiting programme. BMJ Open 2019;9:e031780. doi:10.1136/ bmjopen-2019-031780

- Prepublication history for this paper is available online. To view these files, please visit the journal online (http://dx.doi. org/10.1136/bmjopen-2019031780).

Received 22 May 2019 Revised 19 November 2019 Accepted 20 November 2019

Check for updates

(c) Author(s) (or their employer(s)) 2019. Re-use permitted under CC BY-NC. No commercial re-use. See rights and permissions. Published by BMJ.

For numbered affiliations see end of article.

Correspondence to Dr Samantha Sabo; samantha.sabo@nau.edu

\section{ABSTRACT}

Introduction Emerging evidence suggests community health workers (CHWs) delivering preventive maternal and child health (MCH) interventions through home visiting improve several important health outcomes, including initiation of prenatal care, healthy birth weight and uptake of childhood immunisations.

Methods and analysis The Arizona Health Start Program is a behavioral-based home visiting intervention, which uses CHWs to improve MCH outcomes through health education, referral support, and advocacy services for at-risk pregnant and postpartum women with children up to 2 years of age. We aim to test our central hypothesis that mothers and children exposed to this intervention will experience positive health outcomes in the areas of (1) newborn health; (2) maternal health and healthcare utilisation; and (3) child health and development. This paper outlines our protocol to retrospectively evaluate Health Start Program administrative data from 2006 to 2015, equaling 15576 enrollees. We will use propensity score matching to generate a statistically similar control group. Our analytic sample size is sufficient to detect meaningful programme effects from low-frequency events, including preterm births, low and very low birth weights, maternal morbidity, and differences in immunisation and hospitalisation rates.

Ethics and dissemination This work is supported through an inter-agency contract from the Arizona Department of Health Services and is approved by the University of Arizona Research Institutional Review Board (Protocol 1701128802, approved 25 January 2017). Evaluation of the three proposed outcome areas will be completed by June 2020 .

\section{BACKGROUND}

Over the last decade, the community health worker (CHW) workforce has been recognised by the WHO and several US entities as an evidence-based approach to address health disparities. ${ }^{1-3}$ In the USA, the CHW workforce has gained recognition and visibility, as evidenced by the creation of a US Department of Labor Standard Occupational Classification (21-094) in 2010, to include CHWs as
Strengths and limitations of this study

- A 10-year retrospective observational study of a community health worker home visiting intervention using propensity score matching.

- Size and diversity of mothers in the intervention group (9665) will be matched to one or more characteristically similar mothers in the comparison group.

- Less than $1 \%$ of intervention participants were involved in other home visiting programmes.

- Analysis may have limited external validity for populations who differ along socioeconomic status, race and ethnicity.

a health profession in the Patient Protection and Affordable Care Act. ${ }^{4}$ According to the American Public Health Association (APHA), a CHW is a frontline public health worker who is a trusted member of and/or has an unusually close understanding of the community served. This trusting relationship enables the worker to serve as a liaison/link/intermediary between health/social services and the community to facilitate access to services and improve the quality and cultural competence of service delivery. ${ }^{5}$

Emerging evidence suggests CHWs delivering preventive maternal and child health (MCH) interventions through home visiting improve several important maternal and child outcomes. ${ }^{6}$ Globally, CHW home visiting interventions are associated with several primary prevention efforts that promote the initiation of any, early, and adequate prenatal care, ${ }^{8} 9$ initiation of any and exclusive breastfeeding, ${ }^{70-13}$ reduction of maternal morbidity and perinatal mortality, ${ }^{14}$ and the uptake and completion of childhood immunisations. ${ }^{715}$ In the USA, CHW home visiting interventions are associated with decreased incidence of preterm birth $^{9}$ 16-18 and low birth weight, ${ }^{16-22}$ and 
increases in up-to-date immunisations among newborns and toddlers. ${ }^{23}$ CHWs share the language, socioeconomic status (SES) and life experiences of their clients, making them a fundamental asset to reducing health inequalities among disenfranchised groups. ${ }^{24}$ Moreover, CHWs are recognised as integral contributors in collaborative health-based and community-based teams by improving comprehensive care and addressing the social determinants of health that contribute to health improvements and cost savings. ${ }^{25} 26$

Arizona launched the first iteration of the Health Start Program (HSP) in 1984, when Arizona ranked among the lowest five states for the number of women receiving any or adequate prenatal care. ${ }^{27} \mathrm{HSP}$ is a statewide programme that employs CHWs to engage at-risk, low income, and racially and ethnically diverse mothers and improve maternal and child outcomes. HSP has been managed by the Arizona Department of Health Services (ADHS), Bureau of Women's and Children's Health since 1992. ${ }^{28}$ In 1994, the Arizona state legislature passed the Arizona Children and Families Stability Act, Arizona Revised Statutes (ARS) § 36-697, which formalised and expanded HSP and articulated the purpose, requirements and administration of the programme. HSP is a communitybased outreach programme that identifies, screens, and enrols pregnant women early in their pregnancies and assists them with obtaining early and consistent prenatal care. The programme also provides prenatal and postpartum education, information and referral services, client advocacy, and emphasises timely immunisations and developmental assessments for their children. Since its inception, Arizona HSP's mission has been to educate, support and advocate for families at risk by promoting optimal use of community-based family health care services and education services through the use of community health workers (CHWs) who live in and reflect the ethnic, cultural and socioeconomic characteristics of the community they serve. ${ }^{28}$

\section{Study setting}

Arizona is the sixth largest state in the nation, with a population of 6.8 million people. The state shares an international border with Mexico and is home to 21 federally recognised American Indian Tribes and Nations, making it uniquely racially and ethnically diverse. Arizona has a higher proportion of Latino (30.9\%) and American Indian (5\%) residents compared with the country ( $17.8 \%$ and $1 \%$, respectively) and a comparatively smaller proportion of African American residents (5\% compared with $13 \%$ nationally).$^{29}$

In 2015 , nearly a quarter of the population lived in rural areas, where the poverty rate reached $30 \%$, almost double that of the national poverty rate. ${ }^{29}$ Approximately 20\% of Arizona families with children live below the federal poverty line, compared with $18 \%$ nationally. Poverty disparately affects Arizona's Latino (36\%) and American Indian (46\%) families and children. ${ }^{29}$ Arizona ranks as the fifth highest US state for adult female poverty rate in the country, with more than one quarter of Arizona families headed by single-mother households. ${ }^{29}$ The initial framework for the HSP was developed in the 1980s and 1990s to address the social determinants associated with the steady decrease in the rate of women receiving prenatal care. In the most recent Arizona Title V Maternal and Child Health Needs Assessment (2017), approximately $74 \%$ of pregnant women initiated prenatal care in the first trimester (compared with $61 \%$ in 2015 and $81 \%$ in 2013 ), and $7.9 \%$ had no prenatal care. ${ }^{29}$ There were disparities among mothers by race/ethnicity who received prenatal care, notably American Indian mothers having the highest rates of 'inadequate' prenatal care $(25 \%)$ compared with all women in Arizona $(15 \%) .{ }^{29}$

It is widely recognised that late prenatal care is associated with preterm birth, low birth weight and infant mortality. In 2014, 9\% of babies born in Arizona were premature and $7.2 \%$ were low birth weight. ${ }^{29}$ Historically, low-income mothers have experienced higher rates of premature birth and low birth weight in Arizona ${ }^{30}$ and nationally. ${ }^{31}$ There are also apparent racial disparities for birth outcomes in Arizona. Preterm birth rates are highest among Black (12.2\%), American Indian $(9.4 \%)$ and Latino $(9.2 \%)$ compared with all preterm births $(9.1 \%)$ in the state. Preterm births increase the risk of low birth weight; similar trends persist with the highest rates of low birth weight among Black residents $(10.32 \%)$ compared with White residents $(5.36 \%)$ and the total Arizona population (7.2\%) ${ }^{29}$ Preterm and low birthweight baby delivery costs have been shown to be 25 times more than uncomplicated newborn deliveries. ${ }^{32}$ Although prenatal care and birth outcomes in Arizona have improved over the years, many under-resourced women continue to experience significant challenges and barriers to obtaining healthcare services.

\section{Objectives}

Our goal is to describe the research protocol for a retrospective comparative evaluation to assess the impact of Arizona's HSP, a CHW home visiting perinatal support programme, on multiple maternal, infant and child health outcomes. Broadly, the goal for the study is to meet the federal Home Visiting Evidence of Effectiveness (HomVEE) standard for evidence-based effectiveness. We will use a matched comparison group design that meets the published standard for HomVEE's 'moderate' rating, defined by HomVEE as: '(1) baseline equivalence established on tested outcomes and demographic characteristics and controls for baseline measures of tested outcomes, if applicable; and (2) no confounding factors; must have at least two participants in each study arm and no systematic differences in data collection methods' (note: a 'high' rating is reserved for randomised controlled trials). ${ }^{33}$

\section{Aims}

We plan to objectively test our central hypothesis that mothers and children exposed to HSP during the study period of 2006-2015 will experience positive health 
Table 1 Description of Health Start Program goals, CHW activities (non-exhaustive), predicted client actions, study aims and measurable outcomes. Five maternal and child health goals guide the Arizona Health Start Program CHW activities to support at-risk pregnant and postpartum women and families with children up to 2 years of age. CHWs provide support and services to meet the individual needs of their clients during home visiting sessions that promote self-sufficiency, empowerment, positive health change and improved health outcomes. Our three study aims align with the HSP goals, which we will analyse via the listed outcomes.

\begin{tabular}{|c|c|c|c|c|}
\hline Programme goals & CHW input & Process indicator & Evaluation aims & Measurable outcomes \\
\hline $\begin{array}{l}\text { 1. Reduce the incidence } \\
\text { of very low birthweight } \\
\text { babies. }\end{array}$ & $\begin{array}{l}\text { Prenatal home } \\
\text { visits. Education on } \\
\text { pregnancy, labour, } \\
\text { delivery, nutrition, } \\
\text { inter-conception. } \\
\text { Screening, education, } \\
\text { and assistance for } \\
\text { mood and anxiety } \\
\text { disorders, substance } \\
\text { cessation, and } \\
\text { domestic violence. }\end{array}$ & $\begin{array}{l}\text { Increased knowledge } \\
\text { of and engagement } \\
\text { in pregnancy process } \\
\text { and activities to } \\
\text { promote a healthy } \\
\text { pregnancy. Increase } \\
\text { knowledge of available } \\
\text { services, completed } \\
\text { assistant referrals, } \\
\text { increased access to } \\
\text { services. }\end{array}$ & $\begin{array}{l}\text { Aim 1: assess the } \\
\text { impact of the HSP } \\
\text { on newborn health. }\end{array}$ & $\begin{array}{l}\text { Preterm birth } \\
\text { (gestational age). } \\
\text { Birth weight (birth } \\
\text { weight, low birth } \\
\text { weight }<2500 \mathrm{~g} \text {, very } \\
\text { low birth weight }<1500 \\
\text { g, and small size for } \\
\text { gestational age). } \\
\text { Newborn hospital } \\
\text { length of stay and } 30- \\
\text { day hospital charges. }\end{array}$ \\
\hline $\begin{array}{l}\text { 2. Increase prenatal } \\
\text { services to pregnant } \\
\text { women. }\end{array}$ & $\begin{array}{l}\text { Perinatal home } \\
\text { visits. Assistance } \\
\text { with access and } \\
\text { enrollment to } \\
\text { continuous perinatal } \\
\text { care. } \\
\text { Education on } \\
\text { pregnancy, } \\
\text { labour, delivery, } \\
\text { inter-conception. }\end{array}$ & $\begin{array}{l}\text { Initiate prenatal care } \\
\text { earlier in pregnancy } \\
\text { and attend more } \\
\text { prenatal care visits. } \\
\text { Increased knowledge } \\
\text { of and engagement in } \\
\text { pregnancy process, } \\
\text { delivery options and } \\
\text { activities to promote a } \\
\text { healthy pregnancy. }\end{array}$ & $\begin{array}{l}\text { Aim 2: assess the } \\
\text { impact of the HSP } \\
\text { on maternal health } \\
\text { and care utilisation. }\end{array}$ & $\begin{array}{l}\text { Month prenatal care } \\
\text { initiated. } \\
\text { Total number of } \\
\text { prenatal visits. } \\
\text { Method of delivery (eg, } \\
\text { first-time caesarean } \\
\text { delivery). } \\
\text { Maternal morbidity (eg, } \\
\text { uterine rupture). } \\
\text { Inter-pregnancy } \\
\text { intervals. }\end{array}$ \\
\hline $\begin{array}{l}\text { 3. Reduce the incidence } \\
\text { of children affected by } \\
\text { childhood diseases. } \\
\text { 4. Increase the number } \\
\text { of children receiving } \\
\text { age appropriate } \\
\text { immunisations by } 2 \\
\text { years of age. }\end{array}$ & $\begin{array}{l}\text { Perinatal home visits. } \\
\text { Screening, education } \\
\text { and assistance with } \\
\text { child well-being } \\
\text { services. }\end{array}$ & $\begin{array}{l}\text { Timely completion of } \\
\text { all immunisations for } \\
\text { children. }\end{array}$ & $\begin{array}{l}\text { Aim 3: assess the } \\
\text { impact of the HSP } \\
\text { on child health and } \\
\text { development. }\end{array}$ & $\begin{array}{l}\text { Probability of a child } \\
\text { being on schedule for } \\
\text { immunisations. } \\
\text { Utilisation of } \\
\text { emergency room (ER) } \\
\text { visits and inpatient (IP) } \\
\text { stays at age of } 1,3 \\
\text { and } 5 \text { years. } \\
\text { Any charges } \\
\text { associated with ER } \\
\text { and IP utilisation. }\end{array}$ \\
\hline
\end{tabular}

on the importance

of good nutritional

habits, developmental

assessments and

preventative healthcare.

CHW, community health worker; HSP, Health Start Program.

outcomes in the areas of newborn, maternal and child health (table 1). Specifically, our aims include:

- Aim 1: assess the impact of HSP on newborn health.

- Aim 2: assess the impact of HSP on maternal health and care utilisation.

- Aim 3: assess the impact of HSP on early child health and development.

\section{METHODS: INTERVENTION, PARTICIPANTS AND OUTCOMES HSP intervention}

HSP is significant in that it is one of the longest-standing programmes in Arizona and employs CHWs in 14 distinct Arizona counties to engage at-risk, low-income mothers in order to improve birth outcomes (figure 1). CHWs serve as the primary interventionist for the programme. In 2016, HSP CHWs provided services to 2534 unduplicated clients, conducted 16698 home visits and facilitated 461 classes. ${ }^{28}$ Women are eligible to enrol in HSP if they (1) 


\section{Health Start Program Service Area Map, 2018}

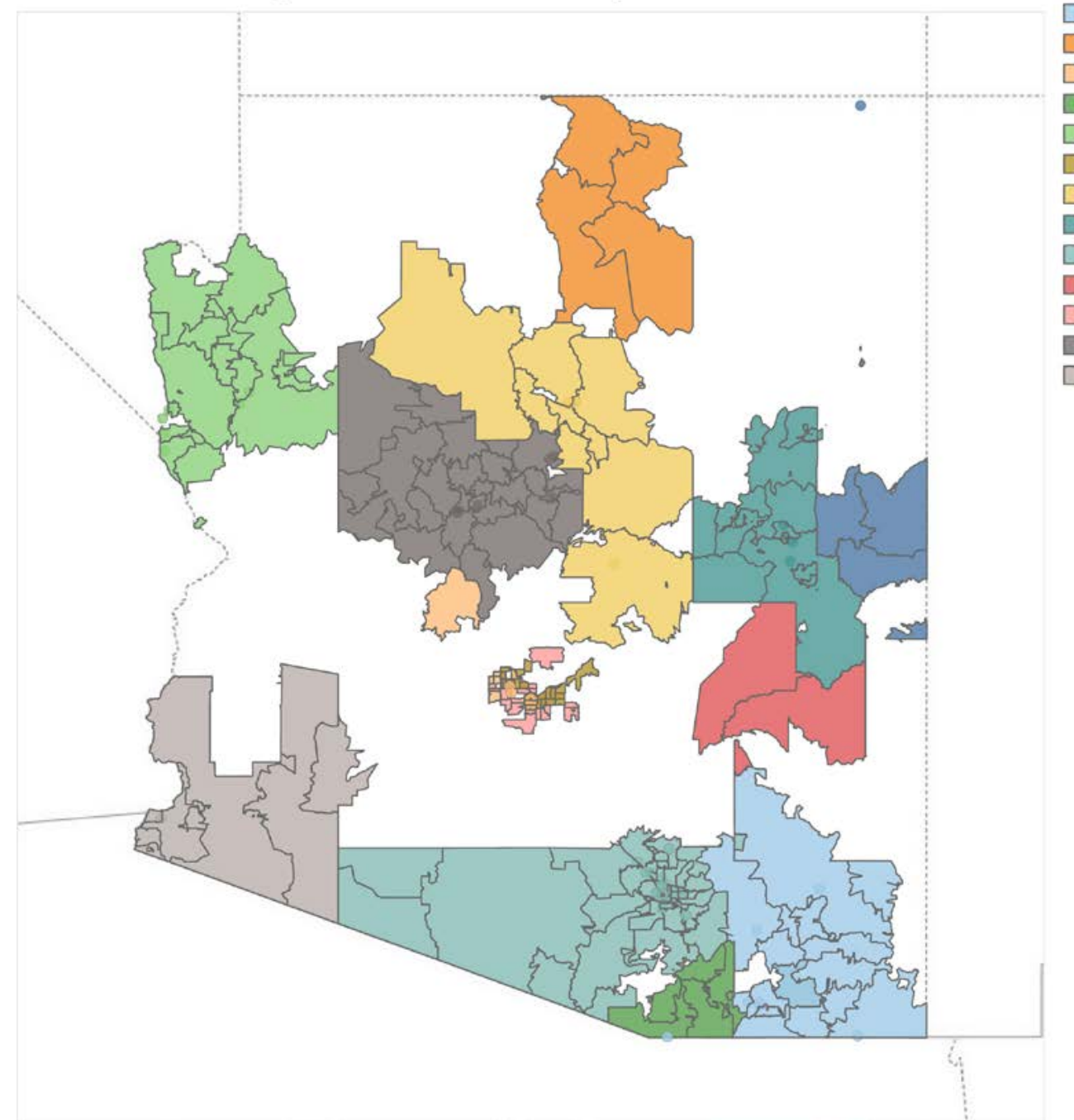

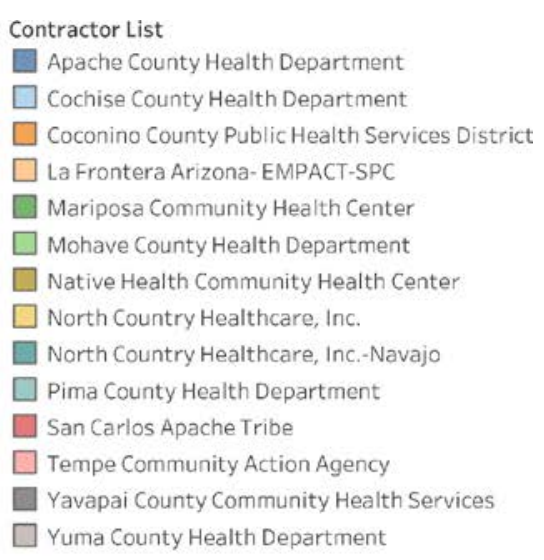
areas within 14 counties across the state. Community health workers conduct regular home visits to under-represented pregnant women and their families in rural and urban communities. Map courtesy of and permission by Arizona Health Start Program, Arizona Department of Health Services. This map is not under copyright.

live in the targeted service area, (2) are pregnant or post partum with a child under the age of 2 years and (3) have one or more social or medical risk factors. Social risks can include but are not limited to: single-parent status, underserved racial or ethnic group, education equal to or less than high school level, annual income less than $\$ 40000$, and Medicaid or no insurance. Medical risks are broad and can include previous preterm birth, low birth weight, chronic disease, high maternal body mass index (BMI) and substance use. Women can be of any age and there are no income requirements to participate.

CHWs connect clients to prenatal care and increase client's continuity of care during and after pregnancy. CHWs identify, screen and enrol eligible women; provide prenatal and postpartum education; provide referral and advocacy services; and emphasise timely immunisations and developmental assessments for children. Although not an exhaustive list, table 1 outlines the primary intervention activities conducted by the CHW. HSP CHW home visits are guided by an asset-based approach and two primary theories of behaviour change, the Trans Theoretical Model and the Social Cognitive Theory. Identifying assets acknowledges and supports the existing strengths and capabilities of individuals and resources to promote community-driven development and positive change. ${ }^{34}$ The Trans Theoretical Model assumes that behaviour modification in individuals is a multistage process in which people move through stages of readiness for change, ${ }^{35}$ and Social Cognitive Theory states that stages occur in the context of reciprocal relationships between the person's environment, their behaviour and their cognition. ${ }^{36}$ CHWs are a community asset and well positioned to support HSP clients; they share both lived experiences and cultural knowledge of the community they serve. The home visiting sessions promote behaviour change through assessment, goal planning, referral, advocacy 
and follow-up activities, coupled with education through meaningful adult learning models. These interactions are designed to encourage personal agency of adult learners to integrate new knowledge and create a cognitive structure that makes sense of their own surroundings and situations. ${ }^{37}$ Through behaviour change theories and adult learning models, the HSP CHWs privilege the co-construction of knowledge among all participants, assume all are co-learners, and encourage critical thinking about self-sufficiency, empowerment, and personal agency related to the five HSP goals (table 1).

\section{HSP CHW core competencies, roles and training}

According to the HSP policy and procedure manual, CHWs must (1) live and work in the service area, (2) reflect the ethnic, cultural and socioeconomic characteristics of the communities they serve, (3) be able to read and write in English, (4) have a high school diploma or General Educational Development and (5) pass a criminal history background check within the Department of Public Safety records to be eligible to work for the statefunded programme. It is highly recommended (though not required) that CHWs have posthigh school training and education in MCH, early childhood development education, family studies, social work, nursing or a closely related field. ${ }^{28}$ Before a CHW can initiate unsupervised outreach or home visits, they must complete 40 hours of training in both the 10 CHW Core Competencies set forth by the CHW Core Consensus Project, ${ }^{38}$ which are recognised by the Arizona state legislature HB 2324 Voluntary CHW Certification, ${ }^{39}$ and the HSP Core Training. ${ }^{28}$ An additional 8 hours of home visit shadowing with a senior CHW are required.

Nationally recognised, the $10 \mathrm{CHW}$ Core Competencies include: (1) Cultural and Systems Mediation; (2) Culturally Appropriate Health Education; (3) Care Coordination and Case Management; (4) Coaching and Social Support; (5) Advocacy; (6) Capacity Building; (7) Direct Service; (8) Individual and Community Assessments; (9) Outreach and (10) Research and Evaluation. ${ }^{38}$ HSP Core Training covers: (1) Essential Health Start Information (HSP basics, visits and community outreach); (2) Communication and Emotional Support; (3) Nutrition and Physical Activity (family nutrition and physical activity, infant nutrition and physical activity); (4) Health Education (healthy pregnancy, prenatal care, discomforts during pregnancy, labour and delivery, postpartum care and family planning, early childhood development and parenting skills, infant health and child health) and (5) Safety (home safety for infants and children, child abuse and domestic violence).$^{28} \mathrm{CHWs}$ are required to complete 12 hours of continuing education per year.

\section{Intervention cohort}

HSP administrative data from 2006 to 2015 is the primary source for identifying the retrospective intervention group. All Health Start clients enrolled during the 10-year observation period will be included in this study if their records are identified and linked from the HSP database to the Vital Records Birth Database (VRBD). Records will be linked based on the mother's date of birth and first name. In order to be a candidate for the HSP study cohort, the mother's date of birth must be an exact match while her first name must be at least $95 \%$ similar, using Jaro-Winkler similarity. ${ }^{40}$ Mother's last name may change due to marriage; therefore, this criterion is not required to identify the intervention cohort. We will obtain the following information for each HSP study cohort mother: a unique identifier (ID), first name similarity percentage, last name similarity percentage, HSP enrollment date, programme closure information (ie, programme completion, reason for closure) and the child's birthdate. Using the process described above, 15576 HSP records were linked to the VRBD.

\section{Intervention cohort sample size}

Our evaluation intervention group will include all HSP participants enrolled within 24 months of the date of birth of the child during 2006-2015. Of the initial 15576 records identified through the HSP-to-VRBD data link, 5911 fall outside of the 24-month (either before or after) HSP enrollment window and will be excluded from all subsequent analyses. The resulting 9665 HSP-associated births constitute the basis of this study (figure 2). Because HSP participants can enrol before or after birth, we will limit the analysis for Aims 1 and 2 (newborn and maternal health outcomes) to those births for which the mother was enrolled during pregnancy. This final criterion results in 6493 HSP-attributed births for the evaluation of Aims 1 and 2. Aim 3 (child health outcomes) will be evaluated using the larger set of 9665 HSP-associated births.

\section{Synthetic comparison group}

A comparison group of women not exposed to the HSP (non-HSP) will be created from all births occurring in Arizona during 2006-2015 (derived from VRBD). After identifying our study population we will use propensity score matching (PSM) to generate a statistically-similar synthetic control group that has, on average, the same observable preprogramme characteristics as the HSP mothers. ${ }^{41}$ The pool of potential comparators will come from all Arizona births that occurred over the study period (2006-2015). This process will be guided by HomVEE standards requiring that the covariates used to balance the treatment and control groups be associated with both treatment status and the outcomes of interest. ${ }^{42}$ Because the HSP eligibility criteria focus on social and medical risks, we will prioritise these types of measures in the PSM model, in addition to characteristics that have been shown to have strong associations with our outcomes of interest in previous empirical and theoretical work.

We will employ radius matching to identify comparison group mothers across the common support region. ${ }^{43} \mathrm{We}$ will use the following measures in the PSM model: mother's birth year, mother's age at birth, county of residence. Additional indicator variables include: child's birth order, 


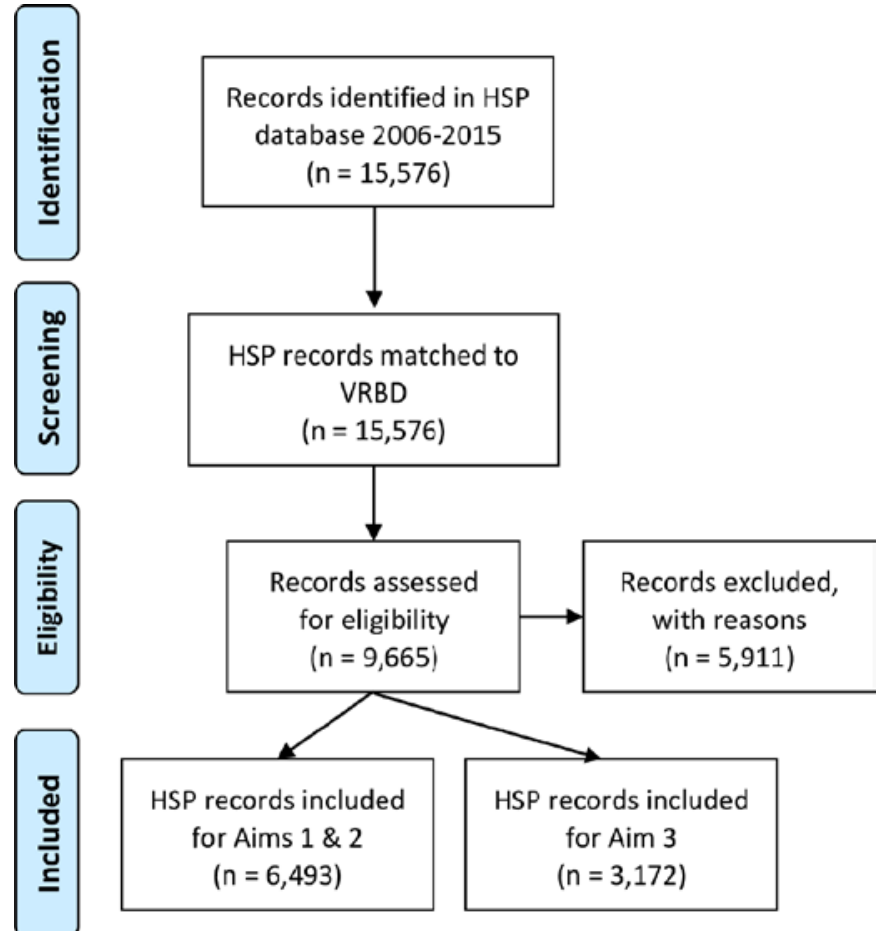

Figure 2 Flow chart of intervention participant inclusion and exclusion criteria. Nine thousand six hundred and sixty-five Health Start Program births constitute the basis of this study. Fifteen thousand five hundred and seventy-six records were initially identified as Health Start Program matches; however, 5911 records were excluded because the child's birth fell outside of the 24-month (either before or after) enrollment window. We evaluate Aims 1 and 2 with a subgroup: records for mothers enrolled in HSP prior to the child's birth (6493 births). We evaluate Aim 3 using the larger set of 9665 HSPassociated births.

maternal educational attainment, health insurance payer (Medicaid being a proxy for low-income status), race, ethnicity, availability of information for the father on the birth certificate, maternal country of birth, previous history of hypertension and median household income by zip code of residence. In addition to these demographic and socioeconomic characteristics, we will restrict potential comparators to mothers within the same fiscal years in order to account for economic conditions and any potentially shifting programme parameters. Imposing withinyear matches will allow us to analyse the programme's efficacy over time by cohort.

Comparison mothers may match to more than one HSP mother, based on the propensity score. Preliminary efforts to identify matches resulted in a potential synthetic comparison group of nearly 23000 non-HSP mothers. Due to the respective sizes of the intervention and comparison populations, lack of statistical power is not a significant issue for this project.

Patients and the public were not involved in the design or planning of the study.
Outcomes

Primary outcomes

HSP is a primary prevention intervention to improve $\mathrm{MCH}$ outcomes among at-risk, racially and ethnically diverse, rural and urban mothers and children of Arizona. We will use four ADHS administrative datasets to evaluate Aims 1-3 including HSP Database, VRBD, Hospital Discharge Database (HDD) and Arizona State Immunization Information System (ASIIS) Database. Aim 1 (HSP impact on newborn health) will be measured by preterm birth, birth weight, and newborn hospital length of stay and associated charges. Aim 2 (HSP impact on maternal health) will be measured by prenatal care initiation and frequency, method of delivery, maternal morbidities and interpregnancy intervals. Aim 3 (HSP impact on child health) will be measured by uptake of age-appropriate immunisations, and emergency room and inpatient encounters and charges (table 2).

\section{METHODS: DATA MANAGEMENT, MONITORING AND STATISTICAL ANALYSIS \\ Data management}

The four datasets that will be accessed for this study will be securely stored and protected through an honest broker. We designated the Center for Biomedical Informatics and Biostatistics' Biomedical Informatics Services at the University of Arizona as the honest broker to facilitate the de-identification, transfer, and management of data, as well as maintain protected health information anonymisation and Health Insurance Portability and Accountability Act (HIPAA) compliance. In this role, the honest broker can identify individuals overlapping between relevant databases, and assign de-identified study codes that would enable cross-linking individuals between the systems.

\section{Data monitoring}

The honest brokers will link the HSP database to the VRBD to generate a comparison group. They will match both the HSP and non-HSP groups to HDD and ASIIS databases using personally identifiable information (eg, name, date of birth, social security number). The honest brokers will create a separate de-identified 'limited data set' for our analyses to compare the mean outcomes of HSP mothers to the comparison group mothers.

\section{Statistical analysis}

The motivation for using PSM to create a synthetic comparison group is to be able to observe the 'counterfactual' to HSP participation, that is, what would have happened in the absence of the programme. We will explore this by comparing outcomes between HSP mothers and those matched to them by the propensity score. More specifically, the average treatment effect generated by PSM will estimate the impact of the programme on the population of both HSP mothers and those who 'look like' HSP 
Table 2 Data sources and outcome measures by study aim. Our retrospective, propensity score-matched observational study pulls data from four sources: HSP Database, VRBD, HDD, and ASIIS. Data were confined to 2006-2015, and serve to evaluate maternal and child health outcomes among at-risk, racially and ethnically diverse, rural and urban mothers and children of Arizona

\begin{tabular}{ll}
\hline Data source (years) & Outcome measures \\
\hline $\begin{array}{l}\text { Health Start Program Data } \\
\text { (2006-2015) }\end{array}$ & Intervention enrollment \\
& Month prenatal care began \\
Vital Records Birth Data & Preterm birth (gestational age) \\
$(2006-2015)$ & Birth weight (birth weight, low birth weight $<2500 \mathrm{~g}$, very low birth \\
& weight $<1500$ g and small size for gestational age) \\
& Month prenatal care began \\
& Total number of prenatal visits \\
& Method of delivery (first-time caesarean delivery) \\
& Maternal morbidity (eg, uterine rupture) \\
Hospital Discharge Data & Inter-pregnancy intervals \\
$(2006-2015)$ & Newborn hospital length of stay and 30-day hospital charges \\
& Utilisation of emergency room (ER) visits and inpatient (IP) stays at \\
& age of 1,3 and 5 years \\
Arizona State Immunization Information & Any charges associated with ER and IP utilisation 3 \\
System & Probability of a child being on schedule for immunisations \\
$(2006-2015)$ &
\end{tabular}

mothers by taking the difference in outcomes between HSP mothers and their matches, and vice-versa.

Our analytic population is of sufficient size to detect meaningful programme effects from low-frequency events, including preterm births, low and very low birth weights, maternal morbidity, and differences in immunisation and hospitalisation rates over a relatively long period. This is also true for specific subgroups served by HSP (eg, Hispanics, American Indian, economically disadvantaged).

Once we establish proper covariate balance between the intervention and matched-control groups, point estimates of the treatment effects will be estimated by comparing outcomes using Stata V.14 software and specifically the teffects command. ${ }^{44}$ Following Abadie and Imbens, ${ }^{45} 46$ this command considers the fact that propensity scores (ie, the parameter that determines the comparison population) are estimated when calculating the standard errors, and thus generates confidence intervals. The propensity scores will not be used as a covariate in traditional regression analysis because it is less effective in forcing baseline equivalence and assumes the relationship between the score and the outcome is linear. ${ }^{41}$

Both the HSP enrollment information and VRBD are administrative data sources, established and maintained for public health monitoring purposes. As such, we do not anticipate missing data to be a significant issue. We assume that such instances (as we find them) are very likely to be the result of human error and not any systematic issues with the data collection and/or reporting processes. Where missing-ness does occur in the variables that make up the propensity score model, we will control for these using dummy variables in place of the missing observations. In the case of missing outcome variables, we will restrict the analytic sample to the non-missing observations, and inspect control variables to verify that there are no systematic differences.

\section{DISCUSSION}

Our evaluation will build on a previous evaluation of HSP conducted by Hussaini $e t$ al, which found that HSP participation was associated with a reduction in the likelihood of a low birthweight outcome. ${ }^{21}$

The Hussaini study used data from 2007 and compared 484 HSP enrollees to almost 5000 non-HSP women; our study compares 9665 HSP enrollees to approximately 23000 non-HSP women spanning 10 years of service. Based on observed covariates, the Hussaini study matching process did not result in baseline equivalence across the two groups. For example, the comparison group was on average 4years older (28.2 vs 24.3) than the HSP mothers. Additionally, Hussaini et al matched to comparison mothers on ex post medical risks, which likely created a bias in favour of finding a positive HSP effect. Our PSM model will generate a comparison group that achieves baseline equivalence of observed covariates. Additionally, we explicitly match on SES variables as required by the HomVEE-published standard for matched comparison group design studies. ${ }^{33}$ Specifically, we match on two individual measures of SES: maternal education and indicators for primary payer for the birth procedure. While these variables satisfy HomVEE's documented standard for measuring SES for group design studies with a 'moderate' rating, we also use the maternal zip code of residence to include a measure of mean household 
income. Finally, we will build on the scope of the original study in two significant ways: (1) by expanding the number of the outcomes considered, including maternal and child outcomes over time, and (2) by performing a number of subgroup analyses that investigate programme impacts based on when in the course of the pregnancy the HSP intervention began, mother's country of origin and maternal age (ie, teen mothers).

\section{Limitations}

The primary limitation is the identifying assumption that selection into the HSP is driven by observable characteristics. This is a limitation common to most PSM analyses. Attenuation bias is a possibility if HSP mothers are incorrectly identified and linked to state birth certificate data. However, the effect of this would be to underestimate (in absolute value) the magnitude of the resulting coefficients, meaning the true effect is likely to be larger (ceteris paribus). In addition, the analysis may have limited external validity for populations who differ along SES, race and ethnicity.

\section{ETHICS AND DISSEMINATION}

Data will be collected by the Arizona Department of Health Services for surveillance and monitoring. Protocol complies with the University of Arizona Biomedical Informatics Service group information security policies including, Information Security Policy (IS-100), Computer and Network Access Agreement (IS-700), Acceptable Use of Computers Policy (IS-701), Electronic Privacy Statement Policy (IS-1000) and Data Classification and Handling Standard (IS-2321) ${ }^{47}$

\section{Dissemination}

On completion of the study, we will initiate major dissemination strategies, including (1) peer-reviewed publications in targeted journals; (2) scholarly presentations at scientific conferences and public health governance meetings; (3) interactive web-based promotional and training materials and (4) strategic informational and planning meetings. In collaboration with ADHS, we aim to submit published journal articles to Mathematica Policy Research for consideration of the HSP as a HomVEE evidencebased practice home visiting model. We will identify local and national forums for dissemination of preliminary results. Findings will be shared with ADHS leadership, Arizona Health Care Cost Containment System (Arizona Medicaid), Arizona Public Health Association, American Public Health Association, MCH-specific conferences and professional forums, the Arizona Association of Federally Qualified Community Health Centers, Association of Health Plans, CHW workforce coalitions, and Maternal, Infant, and Early Childhood Home Visiting.

\section{Author affiliations}

${ }^{1}$ Center for Health Equity Research, College of Health and Human Services, Northern Arizona University, Flagstaff, Arizona, USA
${ }^{2}$ Department of Economics, College of Family, Home, and Social Sciences, Brigham Young University, Provo, Utah, USA

${ }^{3}$ Center for Population Science and Discovery, Community, Environment and Policy Department, University of Arizona, Tucson, Arizona, USA

${ }^{4}$ Center for Biomedical Informatics and Biostatistics, Health Sciences Department, University of Arizona, Tucson, Arizona, USA

${ }^{5}$ Bureau of Women's and Children's Health, Arizona Department of Health Services, Phoenix, Arizona, USA

Acknowledgements We would like to acknowledge the Arizona Department of Health Services (ADHS) and the several ADHS Offices who have supported this partnership, including ADHS Office of Women and Children's Heath and the Health Start Program, ADHS Immunisation Program, and ADHS Vital Statistics. We deeply appreciate their commitment to participating in this study designed to improve the health of women and children of Arizona. We also acknowledge the members of the University of Arizona and Northern Arizona University-ADHS Steering Committee for continued guidance in data sharing and data protection and security. We acknowledge the several Health Start Programs and hundreds of the Health Start Program CHW workforce who have worked tirelessly to improve maternal and child health equity among thousands of mothers, babies and children.

Contributors All authors contributed equally. SS, MB and SR conceptualised the original study protocol. PW and VP conducted data analysis and contributed greatly to the preparation of the analytic methods and data management sections. SS, KM and SR led all protocol writing and editing. MC provided ongoing edits to early and late stage drafts. ADHS will oversee the study and (1) provide input to study design, conduct, data analysis and interpretation of results and review of draft manuscripts; (2) conduct monthly meetings and conference calls to discuss the evaluation impact study, challenges, and barriers and (3) provide Heath Start Program data, documents and data results, programme training and policies manuals as needed.

Funding The Arizona Department of Health Services (ADHS) funds the study, from 1 July 2017 through 30 June 2022. Health Resources Services Administration (HRSA) Maternal, Infant, Early Childhood Home Visiting (MIECHV) provided 17 months of additional federal funding through ADHS during the study period.

Map disclaimer The depiction of boundaries on this map does not imply the expression of any opinion whatsoever on the part of BMJ (or any member of its group) concerning the legal status of any country, territory, jurisdiction or area or of its authorities. This map is provided without any warranty of any kind, either express or implied.

Competing interests None declared.

Patient consent for publication Not required.

Ethics approval Approved by the University of Arizona Research Institutional Review Board (Protocol \# 1701128802), dated25 January 2017.

Provenance and peer review Not commissioned; externally peer reviewed.

Open access This is an open access article distributed in accordance with the Creative Commons Attribution Non Commercial (CC BY-NC 4.0) license, which permits others to distribute, remix, adapt, build upon this work non-commercially, and license their derivative works on different terms, provided the original work is properly cited, appropriate credit is given, any changes made indicated, and the use is non-commercial. See: http://creativecommons.org/licenses/by-nc/4.0/.

ORCID iD

Kelly McCue http://orcid.org/0000-0002-8383-0338

\section{REFERENCES}

1 WHO. Resolution on community health workers to be considered at the upcoming World health assembly. WHO, 2019.

2 Balcazar H, Lee Rosenthal E, Nell Brownstein J, et al. Community health workers can be a public health force for change in the United States: three actions for a new paradigm. Am J Public Health 2011;101:2199-203.

3 Sabo S, Allen CG, Sutkowi K, et al. Community health workers in the United States: challenges in identifying, surveying, and supporting the workforce. Am J Public Health 2017;107:1964-9.

42010 standard occupational classification system: Bureau of labor statistics, 2019. Available: https://www.bls.gov/soc/2010/2010_ major_groups.htm

5 APHA. Community health workers, 2019. Available: https://www. apha.org/apha-communities/member-sections/community-healthworkers 
6 Gilmore B, McAuliffe E. Effectiveness of community health workers delivering preventive interventions for maternal and child health in low- and middle-income countries: a systematic review. BMC Public Health 2013:13:847.

7 Lewin S, Munabi-Babigumira S, Glenton C, et al. Lay health workers in primary and community health care for maternal and child health and the management of infectious diseases. Cochrane Database Syst Rev 2010;94.

8 Poland ML, Giblin PT, Waller JB, et al. Effects of a home visiting program on prenatal care and birthweight: a case comparison study. $J$ Community Health 1992;17:221-9.

9 Williams CM, Cprek S, Asaolu I, et al. Kentucky health access nurturing development services home visiting program improves maternal and child health. Matern Child Health J 2017;21:1166-74.

10 Anderson AK, Damio G, Young S, et al. A randomized trial assessing the efficacy of peer counseling on exclusive breastfeeding in a predominantly Latina low-income community. Arch Pediatr Adolesc Med 2005;159.

11 Furman L, Matthews L, Davis V, et al. Breast for success: a Community-Academic collaboration to increase breastfeeding among high-risk mothers in Cleveland. Prog Community Health Partnersh 2016;10:341-53.

12 Rios-Ellis B, Nguyen-Rodriguez ST, Espinoza L, et al. Engaging Community With Promotores de Salud to Support Infant Nutrition and Breastfeeding Among Latinas Residing in Los Angeles County: Salud con Hyland's. Health Care Women Int 2015;36:711-29.

13 Sandy JM, Anisfeld E, Ramirez E. Effects of a prenatal intervention on breastfeeding initiation rates in a Latina immigrant sample. J Hum Lact 2009;25:404-11.

14 Lassi ZS, Haider BA, Bhutta ZA. Community-based intervention packages for reducing maternal and neonatal morbidity and mortality and improving neonatal outcomes. Cochrane Database Syst Rev 2010:CD007754.

15 Patel AR, Nowalk MP. Expanding immunization coverage in rural India: a review of evidence for the role of community health workers. Vaccine 2010;28:604-13.

16 Coughlin RL, Kushman EK, Copeland GE, et al. Pregnancy and birth outcome improvements for American Indians in the healthy start project of the Inter-Tribal Council of Michigan, 1998-2008. Matern Child Health J 2013;17:1005-15.

17 Brown KK, Johnson C, Spainhower M, et al. Is timing of enrollment associated with birth outcomes? Findings from a healthy start program in Kansas. Matern Child Health J 2017;21:25-31.

18 DeAngelis KR, Doré KF, Dean D, et al. Strengthening the healthy start workforce: a mixed-methods study to understand the roles of community health workers in healthy start and inform the development of a standardized training program. Matern Child Health $J$ 2017:21:65-74.

19 Redding S, Conrey E, Porter K, et al. Pathways community care coordination in low birth weight prevention. Matern Child Health $\mathrm{J}$ 2015;19:643-50.

20 Bouye KH. The resource mothers program: how community health workers can reduce low-birth weight among African-American clients in WIC programs. The Ohio State University, 2005.

21 Hussaini SK, Holley P, Ritenour D. Reducing low birth weight infancy: assessing the effectiveness of the health start program in Arizona. Matern Child Health J 2011;15:225-33.

22 Lee E, Mitchell-Herzfeld SD, Lowenfels AA, et al. Reducing low birth weight through home visitation: a randomized controlled trial. $\mathrm{Am} \mathrm{J}$ Prev Med 2009;36:154-60.
23 Pati S, Ladowski KL, Wong AT, et al. An enriched medical home intervention using community health workers improves adherence to immunization schedules. Vaccine 2015;33:6257-63.

24 Sabo S, Flores M, Wennerstrom A, et al. Community health workers promote civic engagement and organizational capacity to impact policy. J Community Health 2017;42:1197-203.

25 Bovbjerg RB, Eyster L, Ormond BA, et al. Opportunities for community health workers in the era of health reform. The Urban Institute, 2013.

26 Kangovi S, Grande D, Trinh-Shevrin C. From rhetoric to realitycommunity health workers in post-reform U.S. health care. N Engl J Med 2015;372:2277-9.

27 Meister JS, Guernsey de Zapien J. Un comienzo sano: a model prenatal education project. Maternal and Child Health Education Resources 1989;4:1-2.

28 Rumann S. Health start core training manual: a Self-Paced manual for CHW staff 2014.

29 ADHS. Arizona title V maternal and child health services (FY 2018 Application/ FY 2016 annual report) 2017.

30 CDC. Preterm birth and low birth weight in Arizona, 2010. centers of disease control and prevention 2010.

31 HRSA. Child health USA 2013 U.S. department of health and human services health resources and services administration 2013.

32 Russell RB, Green NS, Steiner CA, et al. Cost of hospitalization for preterm and low birth weight infants in the United States. Pediatrics 2007:120:e1-9.

33 USDHHS. HomVEE - Review Process - Producing Study Ratings, 2019. Available: https://homvee.acf.hhs.gov/Review-Process/4/ Producing-Study-Ratings/19/5

34 Kretzmann J, McKnight JP. Assets-based community development. Natl Civ Rev 1996;85:23-9.

35 Prochaska JO, Velicer WF. The transtheoretical model of health behavior change. Am J Health Promot 1997;12:38-48.

36 AJArop B. Social cognitive theory: an agentic perspective 2001;52:1-26.

37 Vallori AB. Meaningful learning in practice. JEHD 2014;3:2334-978.

38 Rosenthal EL, Rush C, Allen C. Understanding scope and competencies: a contemporary look at the United States community health worker field: progress report of the community health worker (CHW) core consensus (C3) project: building national consensus on CHW core roles, skills, and qualities 2016.

39 Arizona HB. 2324, Fifty-third legislature, second Sess 2018.

40 Winkler WE. Overview of record linkage and current research directions. Statistical Research Division, US Census Bureau, 2006.

41 Austin PC. An introduction to propensity score methods for reducing the effects of confounding in observational studies. Multivariate Behav Res 2011;46:399-424.

42 Caliendo M, Kopeinig S. Some practical guidance for the implementation of propensity score matching. J Econ Surv 2008;22:31-72.

43 Dehejia RH, Wahba S. Propensity score-matching methods for nonexperimental causal studies. Rev Econ Stat 2002;84:151-61.

44 Treatment effects/Causal inference | Stata 2019.

45 Abadie A, Imbens GW. Large sample properties of matching estimators for average treatment effects. Econometrica 2006;74:235-67.

46 Abadie A, Imbens GW. Matching on the estimated propensity score. Econometrica 2016;84:781-807.

47 Security UA. Policy and guidance, 2019. Available: https://security. arizona.edu/content/policy-and-guidance 